was a rounded moveable bony prominence (Fig. 1) which was taken to be a dislocated semilunar bone. The wrist appeared to be partially dislocated outwards (Fig. 2). The joints were all very stiff and were forcibly moved under an anæsthetic. Energetic massage and passive movements were then employed and three weeks later, as the dislocated bone was interfering with the functions of the median nerve, it was removed through a small incision. It required little more than a simple incision down to it to effect its dislodgement. When subsequently examined it proved to be the nner half of the scaphoid bone (Fig. 3) and showed a clean cut fractured surface on its outer side. The outer half of the bone apparently remained in articulation with the trapezium and trapezoid. The wound healed by primary union.

The present condition of the limb, eight months after the accident, is as follows. The shoulder-joint is now normal; the elbow-joint allows of flexion to within 10 degrees of the normal and of complete extension. There is absolutely no movement at the radio-ulnar joints and apparently the two bones are firmly fixed together by a bridge of callus (which, however, was too recent at the date at which the skiagram was taken to show a shadow). It has not been considered wise to suggest any operation to ameliorate this condition of affairs, as the patient has a very useful limb and is the subject of granular kidney of some severity. The wrist. joint permits flexion and extension through a range of about 15 degrees and lateral movements through about 25 degrees.

FIG. 2.

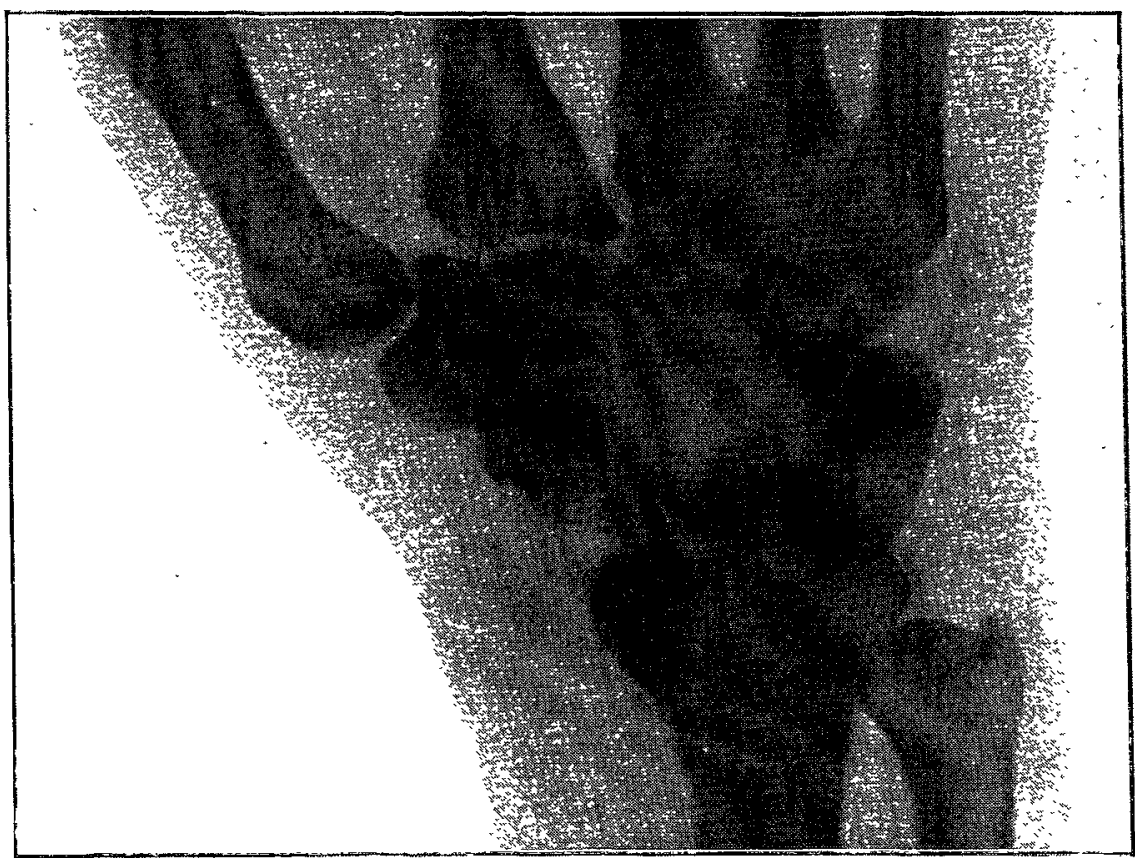

Showing outward dislocation of the wrist.

The movements of the fingers are still not completely restored, especially at the metacarpo-phalangeal joints, but the patient can use them for any but delicate manipulations. This stiffness, which no longer diminishes under the influence of active and passive forcible movements, is at least partly due to the abnormal course of the tendons which have been

Fra. 3.

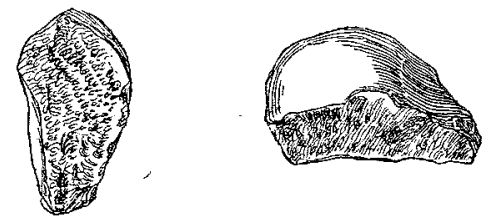

Portion of fractured scaphoid removed.

displaced from their proper grooves on the lower end of the radius. Sensation in the fingers is completely recovered.

It has seemed to me to be worth while to publish this singular accident because in none of the cases recorded and quoted above has the scaphoid bone been cleanly fractured in two whilst a forward dislocation of one of the fragments is especially unusual. Neither have the other recorded cases shown the partial outward dislocation of the rest of fellow. 891 the carpus. No doubt this latter condition has been caused in part by the drawing up of the lower end of the fractured ulna and by the absence of half of the scaphoid bone. The result has, on the whole, been very satisfactory taking into consideration the multiple severe injuries and the unhealthy general condition of the patient. Had passive movements and massage been employed earlier in the case, before the patient returned to London, there can be little doubt that the vicious union between the radius and ulna might have been avoided and the ultimate usefulness of the limb would have been even greater than it actually is.

Gower-street, W.O.

\section{THE USE OF RUBBER GLOVES IN MEDICAL WARDS.}

BX THOMAS WOOD CLARKE, A.B., M.D.,

LATE MEDICAL HOUSE OFFICER, JOHNS HOPKINS HOSPITAI, BALTIMORE; AND RESIDENT PHYSICTAN LAKESIDE HOSPITAL, CLEVELAND, U.SA.

"IN these days of ready invention a glove, I think, might be devised which should be impervious to fluids, and yet so thin and pliant as not to interfere materially with the delicate sense of touch required in these manipulations. One such glove, if such shall ever be fabricated and adopted, might well be sacrificed to the safety of the mother in every labour." 1

The above paragraph from the writings of Sir Thomas Watson, published towards the end of the firt half of the last century, is a strong plea for the use of gloves in the prevention of puerperal sepsis and is probably the first mention of gloves in medicine. Indiarubber was then, however, in its infancy as an article of commerce and it was not until nearly 50 years later that Halsted, in 1889 , first made use of rubber gloves in his surgical technique ${ }^{2}$ In 1894 the subject became of general interest and by 1900 most European and American surgeons were using gloves in their practice. So much has been written during the past ten years by such men as Mikulicz, ${ }^{3}$ Manteuffel, ${ }^{4}$ Keen, ${ }^{5}$ Robb, ${ }^{8}$ McBurney, ${ }^{7}$ and hosts of others, and the use of gloves has become so extensive in surgery, obstetrics, and pathology, that there is little left to be said upon the subject from the point of view of these branches of the profession. The value of rubber gloves to the medical man, however, is so slightly appreciated, and their use in medical wards is so rare, that it may not be out of place to discuss the subject from the physician's stand point. As to the literature, I have been able to find but one article which with any adequacy covers the medical aspect of the rubber glove, and this, though most excellent in itself, is inaccessible in London and was obtained only through the courtesy of the author, M. Faure of Paris. ${ }^{8}$

The subject may well be discussed under three headings: the protection of the patient from the physician, the protection of the physician from the patient, and the protection of the patiest from his

It not infrequently occurs in the wards of a hospital that the so-called " medical operations" must be performed. These are often undertaken with great trepidation, owing to the fear of sepsis, a fear well grounded, especially if the operator is a man who has done little or no surgery for several years. It is, indeed, a serious matter to introduce

1 Sir Thomas Watson: Lectures on the Principles and Practice of Physic, I.ondon, 1843. vol. ii.. p. 349

2 W. S. Halsted : Johns Hopkins Hospital Reports, vol. ii., No. 5,

3 Mikulicz : Revue de Chirurgie, Paris, 1898, vol. xviii., p. 943.

4 Manteuffel : Centralblatt fïr Chirurgie, 1897, vol. xxiv., p. 553. 5 Keen - Annals of Surgery. 1898, vol. xxvii., p. 22

Robb: Cleveland Medical Gazette, 1900-01, vol. Xv., p. 553.

7 MeBurney : Annals of Surgery, 1898, rol. xxviii., p. 108.

- Faure: Revue Gécérale de Clinique et de Thérapeutique, Paris, 1903, vol. xvii., p. 737 . 
infectious material from the outside into a punctured wound and then to seal up the outlet, and more especially so when this wound leads into the pleural or peritoneal cavity, as in the case of a paracentesis, into the meninges in lumbar puncture, or directly into the circulatory system in intravenous injections. Any procedure which will tend to lessen the danger in these operations is surely an advance in the facility of definite diagnosis of doubtful cases and of consequent treatment, and anything which lessens the liability to sepsis is such a procedure. The advantage of rubber gloves in operations, their ease of sterilisation, their impermeability to bacteria, and their immaterial interference with touch and action, have been so thoroughly proved by the surgeons that they must be accepted as facts requiring no further defence. And so, inasmuch as each year the rubber glove comes to be considered less and less as a merely desirable adjunct to surgery and more and more as a necessary one, just so rapidly its value should be appreciated in the medical ward when the physician has to expose his patient to the dangers of sepsis. During the past two years, while in charge of an active medical service in a general hospital, I have made use of rubber gloves in performing infusions, venesections, intravenous injections, paracenteses, and lumbar punctures, and if I have no proof of having saved life by their use I have at least the satisfaction of feeling that $I$ have done everything in my power to eliminate danger.

Passing on from the safety of the patient we come to the protection of the physician and nurse in handling certain classes of cases, a subjzct which has an resthetic as well as a practical aspect. Cises of "obstetrical chancre," though happily not common, are of sufficiently frequent occurrence and grave consequence to deserve consideration, especially as the disease may be contracted when no suspicion of danger exists. The use of rubber gloves, worn during all vaginal and rectal examinations is obviously a great safeguard against syphilitic infection and their value depends entirely upon their practicability. By many it is contended that the gloves so materially interfere with the sense of touch that their use is a hindrance to the physician and a danger to the patient and for this reason should not be worn. My experience, however, leads me to differ from this opinion. By the use. of a glove sufficiently thin, pliant, and. well-fitted, with the tingers short enough to prevent wrinkling at the tips, the interference with the tactile sense is so slight that it may be largely disconsidered. At first a finger trained to direct palpation may feel strange when encased in a glove but in a short time the brain learns to allow for the slight additional resistance and then as much can be made out with a glove as without one. In fact, I once heard an eminent surgeon say that he had used gloves for so many years that he thought if he attempted to operate without them that things would feel unnatural. Though it would probably take the average physician some time to reach this Utopian stage he can approximate it in a remarkably short time and if confused in his early efforts he can with ease remove the glove and confirm his findings with the naked finger. Besides preventing the danger of infection the use of the glove obviates entirely those disagreeable clinging odours, which at times no amount of washing will remove, which make the physician, sometimes for hours after an examination, revolting to himself, burdensome to society, and obnoxious to his patients. The nurse, too, may be protected against infection by the use of a heavier glove to be worn while handling specific cases, when giving inunctions, douches, enemata, \&c.

It may seem at first sight that the use of thin, fragile gloves for the above purposes would be a great additional trouble and expense. With a moderate amount of care, however, on the part of the physician one pair of gloves of good quality will last for many weeks or even months, thus reducing the expense to a minimum. The technique of caring for the gloves consists of washing them immediately after use, boiling for a few minutes, drying thoroughly, care being taken to prevent burning, and powdering inside and out with talcum powder. This takes but a very few minutes of a nurse's time and so prepared the gloves keep in good condition for a long time and are ready for use at a moment's notice for examinations, or they may be boiled with the instruments when surgical cleanliness is required.

There now remains to be considered the protection of the

Fox and Schumann: Amerivan Journal of the Medical Sciencer, 1905, vol. cxxx., p. 636 . patient from his fellows. Though most general hospitals have adequate provisions for the isolation of contagious diseases in many those cases classed as infectious must be treated in the open wards. It is in the latter contingency that the rubber glove is of value. This is especially true in diseases where the infection is carried through the excreta. The thorough disinfection of the hands of a nurse in a busy ward, whenever she gives a bedpan to a typhoid patient or changes linen soiled by excreta, is a difficult procedure and the most conscientious nurse may become a source of danger to other persons. If this is true of adult patients it is even more striking in a children's ward during the summer months, when the duties of the overworked nurses consist largely of changing the soiled napkins of diarrhcea cases and of preparing the children's nourishment. The infectiousness of entero-colitis, whether due to Shiga's bacillus or to some other organism, is sufficiently well proved and enough ward epidemics of diarrhoea have occurred, where the original supply of milk has been of undoubted purity, to make it evident that contamination may take place in the ward. The thought is not a pleasant one of a nurse, with hands more or less fouled from a fluid stool, giving a biscuit or a bit of bread-and-butter to a child. Even the knowledge that she has washed her hands between times and "disinfected" them by a perfunctory dip into a carbolic acid solution does not make the thought entirely agreeable.

During a house epidemic of typhoid fever at the Lakeside Hospital in Cleveland in January, 1904, a committee was appointed, of which I was a member, to investigate the cause of the outbreak and to take necessary steps to check it. Among other changes recommended was the use of rubber gloves by the nurses whenever handling bedpans, soiled linen, or napkins of typhoid and enteritis patients. The technique was made as simple as possible. A large dish of carbolic solution was placed in each lavatory and in it were kept several pairs of heavy strong rubber gloves, similar to those commonly worn by pathologists. To facilitate rapid changes they were several sizes larger than the hands of the nurses. When a bedpan was called for, or a napkin to be changed, the nurse rinsed a pair of the gloves in water and slipped them on her hands, a procedure requiring but a few seconds. The gloves were kept on until the stool was taken to the lavatory, broken up with a glass rod, if solid, and carbolised. They were then returned to the carbolic solution and were soon ready to be used again. The objection at first raised of lack of time to carry out these innovations was soon dropped and within a few days the nurse began to look upon the glove as a boon instead of a hardship. Not only was time saved by the reduction of the amount of scrubbing required but it was soon found that the hands, previously kept cracked and sore by the frequent soaking in carbolic acid, were again resuming their normal healthy condition and were kept clean with less trouble and without pain. This procedure has been used constantly for the past two years and has giren complete satisfaction.

In one other condition I would suggest the possible value of this use of the rubber glove, though $I$ do not speak from personal experience. This is in that unhappy epidemic which occasionally breaks out in a children's ward of valvovaginitis, due to an organism resembling the gonococcus, and which spreads rapidly to every female child in the ward and may even require the temporary exclusion of girl patients. As this affection attacks infants as well as older girls bedpan infection can be excluded. It is possible, but seems un. likely, that air contagion is the cause. It is not pleasant to think of the hand of the nurse as the means of transmission but this possibility must not be forgotten. If this be the cause, surely the precautions taken with cases of typhoid fever and diarrhoea may be so modified as to be of value in combating this disease as well.

It is not the purpose of this paper to cover exhaustively the many uses of rubber gloves but rather to point out a few ways in which they have been of value, in the hope that the physician may not fall too far behind the surgeon, the obstetrician, and the pathologist in his appreciation of their worth. The paper is written from the standpoint of the hospital ward, but much of the contents apply equally well to private practice.

I wish to express my thanks to Dr. George W. Crile, Dr. Edward F. Cushing, and Miss Katherine Lilly of Cleveland to the first for the reference to Sir Thomas Watson; to the second for many of the ideas here incorporated; and to the last for the exact technique of the care of the gloves at the Lakeside Hospital and for her opinion of them from the nurses' standpoint. 